\title{
Synthèse de polyglycérols linéaires et cycliques. Tensioactifs polyglycérylés : synthèse et caractérisations
}

\section{Synthesis of linear and cyclic polyglycerols. Polyglyceryled surfactants: synthesis and characterization}

Oléagineux, Corps Gras, Lipides. Volume 9, Numéro 2, 155-62, Mars - Juin 2002, Fondamental

Auteur(s) : Catherine DEBAIG, Thierry BENVEGNU, Daniel PLUSQUELLEC, ETSCO, 50, rue Michelet, BP 808, 49000 Angers Cedex 01.

Author(s) : Catherine DEBAIG, Thierry BENVEGNU, Daniel PLUSQUELLEC

Résumé : Le glycérol est l'un des principaux sous-produits de l'oléochimie. Un des axes de recherche pour valoriser ce co-produit demeure la production de polyglycérols et d'esters de polyglycérols, tensioactifs non ioniques. Cependant, ceux-ci sont généralement préparés industriellement dans des conditions drastiques (milieu caustique et températures élevées), générant des mélanges complexes d'oligomères de composition moléculaire mal définie. C'est dans ce contexte qu'un programme européen a été initié dans le but de préparer des échantillons standard et d'établir des corrélations structure/activité (analyse, performances, environnement) par une étude plus fondamentale sur les oligomères du glycérol et leurs esters. Notre travail a consisté à mettre au point des voies d'accès à des oligoglycérols linéaires et cycliques, de degré 2 à 5 , ainsi que des monoesters et monoéthers d'acides gras, de structure parfaitement définie, en privilégiant des synthèses courtes et efficaces pour obtenir des échantillons de plusieurs grammes. Les stratégies pour les structures linéaires reposent dans un premier temps sur des synthèses convergentes impliquant l'ouverture régiospécifique d'époxydes. La préparation de dimères cycliques du glycérol repose sur des réactions d'iodocyclisation, alors que les oligomères cycliques de degré 3 à 5 ont été préparés via la condensation de deux fragments linéaires. Dans un deuxième temps, le choix de groupes protecteurs orthogonaux et de réactions sélectives a permis de synthétiser une gamme de molécules amphiphiles de structure définie, en faisant varier aussi bien la tête polaire (nombre d'unités glycérol impliquées) que la chaîne hydrophobe (longueur, insaturation, position sur la tête polaire, liaison chimique ester ou éther).

Summary : Glycerol is one of the main by-products of oleochemistry. In order to develop the possible uses of this by-product, research can focus on the production of polyglycerols and their non-ionic surfactants. However, they are usually prepared under drastic conditions (caustic media and high temperatures) leading to complex mixtures of undefined oligomers. In this context, a European Program started to prepare authentic standards of oligomers of glycerol as well as corresponding surfactants and to establish relations between structures and activity. This work describes a new means of access to authentic standards of linear and cyclic oligoglycerols and their mono-esters or mono-ethers with well-defined structures and determined degrees of polymerisation from 2 to 5 , through short and efficient syntheses allowing further scaling up. On the one hand, linear oligomers were obtained by means of a convergent approach based on regioselective opening of epoxides. lodocyclisation reactions involving unsaturated alcohols permitted to build cyclic diglycerols, whereas larger cycles were prepared thanks to the condensation of two linear fragments. On the other hand, the use of orthogonal protective groups and of selective reactions yielded a large range of amphiphilic molecules bearing different kind of fatty chain (length, unsaturations, position on the polar head, ester or ether bond). 
Mots-clés : polyglycérols, tensioactifs, standards, méthode de synthèse.

Keywords : polyglycerols, surfactants, standards, synthetic methods.

\section{ARTICLE}

La plus grande partie de la production du glycérol tient peu de compte de la demande du marché, puisqu'il provient essentiellement de la transformation d'huiles, de graisses et autres acides gras. L'offre de glycérol, principal produit secondaire de l'oléochimie, augmente donc avec la production d'acides gras et d'alcools gras ou encore de biocarburants issus de procédés de transestérification d'huiles végétales (huile de colza, par exemple) par le méthanol, procédés en plein développement. Cependant, le cours du glycérol participe à la rentabilité de ces filières de lipochimie et il est donc primordial de valoriser ce co-produit en développant de nouvelles utilisations [1].

Une voie de valorisation utilise naturellement le caractère hydrophile de ce triol et de ses polymères comme composant de tensioactifs non ioniques, susceptibles d'être utilisés comme émulsifiants dans l'alimentation, les cosmétiques ou les produits pharmaceutiques [2]. Toutefois, malgré le nombre important d'applications potentielles, une des limitations au développement des polyglycérols et des tensioactifs associés est d'ordre technique [3]. Il existe en effet peu de données analytiques et environnementales les concernant. Ceux-ci sont généralement préparés industriellement dans des conditions drastiques (milieu caustique et température élevée) qui conduisent à des mélanges d'oligomères où le nombre d'unités glycérol, la structure (linéaire, ramifiée ou cyclique), le nombre de substitutions sur la chaîne polyglycérol et les isomères obtenus sont difficilement régulés. Leur composition moléculaire peut se révéler complexe comme l'illustre la figure 1 avec l'exemple des diglycérols, et il est évident que le nombre d'isomères possibles croît exponentiellement avec le degré d'oligomérisation.

Jusqu'à présent, la multiplicité des structures produites ainsi que l'absence d'étalon de référence a véritablement freiné les méthodes d'analyses chromatographiques [4-7]. Les études des propriétés physicochimiques ont également été uniquement réalisées sur ces mélanges de dérivés d'oligoglycérols [8,9]. Afin de permettre de produire des mélanges plus ciblés et de mieux cerner les propriétés physico-chimiques et l'impact environnemental de chacun de leurs composants, une étude plus fondamentale sur les oligomères du glycérol et leurs esters est apparue nécessaire. Nul ne connaît en effet l'influence que peuvent avoir les structures cycliques sur l'irritabilité dans des utilisations en cosmétique, par exemple. Ainsi, les préparations industrielles pourraient être orientées en sélectionnant les synthèses chimiques conduisant aux isomères les plus actifs et les moins nocifs. Dans ce contexte, un programme européen FAIR a été initié en 1997 [10] dans le but de déterminer les corrélations qui peuvent exister entre les structures chimiques des oligoglycérols et de leurs dérivés tensioactifs et leurs performances, leur impact sur l'environnement ou leur pouvoir irritant sur la peau et les muqueuses. Cela a nécessité dans un premier temps la synthèse d'échantillons de structure parfaitement définie pour constituer les standards qui sont à la base de ces études. Les travaux présentés ici concernent la mise au point de voies d'accès à des oligoglycérols linéaires et cycliques, de degré 2 à 5 ainsi qu'à une gamme de monoesters et de monoéthers d'acides gras, de structure parfaitement définie. 


\section{Préparation d'oligoglycérols linéaires}

Les synthèses connues de polyglycérols [11] mènent, à quelques exceptions près [12-14], à des mélanges de différents oligomères. Afin d'obtenir des échantillons de structure parfaitement définie, la stratégie choisie implique des synthons clés et des synthèses convergentes pour construire des oligoglycérols linéaires comportant un nombre défini d'unités glycérol. L'objectif, à terme, étant non seulement d'utiliser ces composés comme tête polaire de molécules tensioactives, mais aussi de réaliser différentes études (propriétés physico-chimiques, biodégradabilité, mesure du pouvoir irritant sur les muqueuses, etc.), les méthodes de synthèse se devaient d'être rapides et faciles à mettre en oeuvre afin de disposer de plusieurs grammes d'échantillon.

La stratégie (figure 2) repose sur la synthèse, dans une première étape, de précurseurs linéaires, dimères ou trimères du glycérol, comportant un ou deux cycles oxiranes. L'ouverture régiospécifique de ces époxydes, sur la position la moins encombrée, par un alcool primaire monomérique tel le solkétal (acétamide du glycérol), conduit alors à des oligomères linéaires de degré défini [15]. L'extension de la chaîne polyglycérylée est ainsi contrôlée et peut s'effectuer par une seule extrémité ou par les deux (gain de deux unités glycérol).

Le précurseur 1 est facilement obtenu en une seule étape, par oxydation de la double liaison de l'éther allylique de glycidyle 4, composé commercial. Le monoépoxyde $\mathbf{2}$ résulte d'une éthérification de Williamson entre le solkétal et l'épichlorhydrine [16]. Enfin le bis-époxyde 3 est accessible en trois étapes à partir de l'épichlorhydrine et de l'alcool allylique via une éthérification puis la protection de l'hydroxyle central par un éther benzylique, suivi de l'oxydation des deux doubles liaisons par l'acide $m$-chloroperbenzoïque ( $m C P B A$ ) (figure 3).

Afin de préparer les oligomères linéaires cibles tri-, tétra- et pentaglycérol ( $c f$. l'exemple du pentaglycérol 9 figure 4), nous avons ensuite étudié la réaction classique d'ouverture régiospécifique des oxiranes des composés 1, $\mathbf{2}$ et $\mathbf{3}$ en milieu basique. Notre choix s'est porté sur l'utilisation d'un excès de solkétal dans un milieu hydro-organique constitué d'un solvant aqueux à $50 \%$ d'hydroxyde de sodium et de $n$-hexane, en présence d'un catalyseur de transfert de phase (bromure de tétrabutyl ammonium, TBAB, ou Aliquat ${ }^{\circ}$ ). L'intérêt majeur de la catalyse par transfert de phase [17] réside dans sa facilité de mise en œuvre. C'est un procédé simple et économique en solvant qui constitue une approche pratique et efficace permettant d'éviter les conditions rigoureuses habituellement nécessaires pour la synthèse d'éthers [18].

\section{Préparation d'oligoglycérols cycliques}

Bien que les structures cycliques ne soient présentes que de façon minoritaire dans les mélanges industriels, il est important de les étudier à part entière puisque leurs effets potentiels en termes de biodégradabilité et de toxicité sont quasi inconnus. Ces composés peuvent résulter de réactions de condensation intramoléculaire à partir d'oligomères linéaires ou ramifiés [5]. Ils sont généralement décrits comme des sous-produits minoritaires des réactions de polymérisation, dont la présence n'est pas souhaitée et que les producteurs cherchent plutôt à minimiser. Contrairement aux oligomères linéaires, un grand nombre d'isomères peut être envisagé à chaque degré d'oligomérisation pour les structures cycliques (cf. figure 1 dans le cas des dimères).

Dans ce travail, nous avons sélectionné des cycles réguliers (figure 5) de type 1,4-dioxane bis-substitué, ou pour un degré d'oligomérisation supérieur à 2, des structures correspondant au cycle le plus étendu, c'est-àdire ne comportant que des hydroxyles secondaires libres, structures qui pourraient provenir de la condensation des deux extrémités de l'oligomère linéaire correspondant. 
L'étape clé de la synthèse des structures $p$-dioxane $\mathbf{1 0}$ et $\mathbf{1 1}$ est une réaction d'iodocyclisation de type 6-exotrig impliquant des alcools insaturés convenablement choisis. Les alcools insaturés $\mathbf{1 5}$ et $\mathbf{1 7}$ (figure 6) sont accessibles en quelques étapes à partir de produits de départ commerciaux. L'optimisation des conditions de cyclisation (choix du solvant, du donneur d'halonium) a permis d'obtenir les structures cycliques $\mathbf{1 6}$ et $\mathbf{1 8}$ avec des rendements voisins de $50 \%$, tout en limitant l'apparition des produits secondaires : composés di-iodés 19 et $\mathbf{2 0}$ issus de l'addition électrophile de l'iode sur la double liaison ou dioxolane $\mathbf{4 0}$ résultant d'une iodation allylique suivi d'une cyclisation intramoléculaire. Une fois le cœur $p$-dioxane construit, une suite de réactions de protection-déprotection conduit aux deux diglycérols cycliques 10 et 11 [15].

Dès que l'on considère un degré d'oligomérisation supérieur à 2, les cycles peuvent être considérés comme des éthers-couronnes polyfonctionnalisés. La stratégie de synthèse des tri-, tétra- et pentaglycérols cycliques $(12,13,14)$ repose sur la condensation de deux fragments linéaires bifonctionnels : un diol, monomère, di- ou trimère linéaire du glycérol $(\mathbf{2 3}, \mathbf{2 4}, \mathbf{2 5})$ et un diglycérol linéaire comportant deux groupes partants. Les diols sont issus de réactions de protection-déprotection successives impliquant des intermédiaires décrits lors des synthèses des oligoglycérols linéaires. La présence des groupements benzyles, encombrants, sur les hydroxyles secondaires (figure 7, p. 159) nous a fait préférer les triflates aux groupes tosylates, très souvent employés dans ce type de réaction [19-23], mais présentant une absence totale de réactivité lorsqu'ils sont appliqués à nos substrats.

L'alkylation des diols $\mathbf{2 3}, \mathbf{2 4}$, et $\mathbf{2 5}$ avec le bis-triflate $\mathbf{2 2}$ en présence d'hydrure de sodium et sans utiliser de condition de haute dilution a fourni les macrocyles perbenzylés avec un rendement de $35 \%$ pour chaque oligomère [15].

\section{Tensioactifs polyglycérylés}

Les tensioactifs polyglycérylés commercialisés sont généralement des mélanges statistiques avec un nombre d'unités glycérol et un degré d'estérification plus ou moins contrôlé mais qui peut rester aléatoire (nombre de chaînes grasses et positions) [11]. En effet, si on considère une tête polaire telle que le triglycérol linéaire, on constate que de nombreuses molécules amphiphiles peuvent être construites (figure 8, p. 159) en intégrant une ou plusieurs chaînes grasses (potentiellement jusqu'à cinq) et en faisant varier sa (ou leur) position sur le squelette symétrique du triglycérol. Afin de préparer un panel de molécules représentatif pour établir par la suite des corrélations entre structure chimique, propriétés tensioactives, pouvoir irritant et biodégradabilité, nous avons cherché à greffer une seule chaîne grasse sur des positions bien définies des différents oligoglycérols synthétisés, en apportant des modulations par le choix des chaînes hydrophobes : longueur $(C 12, C 16)$, insaturation (C16:0, C16:1) et par la liaison chimique impliquée (ester ou éther) [24, 25].

Quelle que soit la tête polaire (di- à pentaglycérol linéaire), les stratégies de synthèse reposent sur l'utilisation de groupements protecteurs orthogonaux et de réactions d'estérification sélectives. Afin d'illustrer ce propos, considérons à nouveau le triglycérol linéaire, avec ses trois positions accessibles : centrale, terminale et interne (figure 9, p. 160). Grâce au composé 29, précurseur direct du triglycérol linéaire comportant deux groupements acétonides qui bloquent les positions internes et terminales, seul l'hydroxyle central est accessible à une estérification ou une éthérification directe en présence respectivement d'un chlorure d'acide ou d'un alcool gras activé sous la forme d'un tosylate. Les conditions douces de déprotection (résine acide) réduisent les risques d'hydrolyse ou d'isomérisation des chaînes esters. 
Le greffage d'une chaîne grasse sur un des hydroxyles primaires du triglycérol nécessite a priori la protection préalable des hydroxyles voisins. La position centrale est aisément bloquée à partir de l'intermédiaire 29 et le tétraol 32 résulte de son hydrolyse acide. La désymétrisation de ce polyol s'étant avérée problématique, une monoestérification sélective a été envisagée par le biais d'un réactif acylant de type thiazolidine-2-thione [26]. L'addition lente d'un léger excès de cet agent acylant dans le THF en présence d'une quantité catalytique d'hydrure de sodium permet d'estérifier préférentiellement les hydroxyles primaires, plus acides. Le monoester produit est ensuite séparé des co-produits minoritaires de multiestérification par chromatographie sur colonne de gel de silice. Une hydrogénolyse dans des conditions classiques donne alors accès aux amphiphiles ciblés. La spectroscopie RMN, à l'aide des séquences complémentaires de type DEPT, COSY et HETCOR nous permet d'identifier la structure des monoesters synthétisés (tableau 1) et d'évaluer leur pureté. Ainsi, pour les chaînes à 16 atomes de carbone (C16:0 et C16:1), les monoesters isolés sont des mélanges inséparables par colonne chromatographique des composés $\mathbf{3 3}$ et $\mathbf{3 4}$ (respectivement 15 et $10 \%$ de $\mathbf{3 4}$ ), ce dernier portant la chaîne grasse sur la position vicinale.

Le greffage sélectif d'un monoéther sur cette position terminale a été mené à bien en utilisant une stratégie impliquant l'ouverture régiospécifique de l'oxirane du précurseur $\mathbf{3 5}$ avec le dodécanol dans des conditions de transfert de phase. L'intermédiaire $\mathbf{3 5}$ est accessible en 3 étapes à partir du synthon monoépoxyde $\mathbf{2}$ (figure 9 , p. 160).

Pour atteindre sélectivement la $3^{\mathrm{e}}$ position - interne -, nous avons choisi de protéger les hydroxyles primaires en ouvrant simultanément les deux époxydes du précurseur $\mathbf{3}$ avec l'alcool benzylique. Ainsi, une estérification du diol obtenu conduit à un mélange des mono- et diesters d'acide gras, facilement séparables sur colonne de gel de silice. Lors de l'étape de déprotection, ou même dans des conditions faiblement acides, nous avons observé une migration de l'ester gras sur la position primaire, phénomène réduit par l'utilisation de l'acétate de palladium comme catalyseur d'hydrogénolyse.

Les stratégies décrites ci-dessus (groupes protecteurs orthogonaux et estérification sélectives) ont été appliquées et parfois modifiées pour préparer des amphiphiles à partir des différentes têtes polaires : diglycérol, tétra- et pentaglycérol linéaires. Le tableau 2 (p. 161) récapitule l'ensemble des structures synthétisées [27]. Dans le cas des diglycérols cycliques, les monoesters ont été construits à partir des intermédiaires iodés $\mathbf{1 6}$ et $\mathbf{1 8}$ en transformant la liaison iodométhyle via une substitution nucléophile de l'iodure avec l'anion carboxylate de l'acide laurique, formé in situ à partir d'acide laurique et de carbonate de potassium (figure 10, p. 161).

\section{Exploitation des molécules synthétisées [10]}

L'ensemble des échantillons préparés, oligomères libres du glycérol et amphiphiles associés, constituent des standards qui pourraient être utiles pour analyser des milieux polyglycérylés complexes. Dans le cadre de ce travail, en effet, les profils HPLC de ces composés purs ont été déterminés grâce à une même phase stationnaire (carbone graphitisé poreux) et un détecteur à diffusion de lumière, en ajustant les systèmes d'éluant pour les oligoglycérols (eau/acétonitrile) et pour les amphiphiles (méthanol/dichlorométhane) [28, 29].

D'autre part, l'étude des propriétés physico-chimiques a montré que les tensioactifs polyglycérylés, de structure parfaitement définie, présentaient un comportement typique de tensioactifs non ioniques, avec toutefois des tensions interfaciales entre l'eau et le $n$-décane particulièrement basses, ce qui implique de bonnes propriétés émulsifiantes. On a pu noter dans le cas des structures cycliques un effondrement du 
volume de mousse produite. L'impact de ce type de composé sur l'environnement reste assez faible puisque la plupart des molécules testées se sont révélées totalement biodégradables, à l'exception des composés 10 (cœur cyclique) et $\mathbf{3 1}$ (monoéther en position terminale du triglycérol). Enfin, les mesures d'irritation de ces substances sur les muqueuses ont montré que les oligoglycérols libres, quelle que soit leur structure, avaient un faible potentiel irritant, y compris le diglycérol cyclique. Les résultats ont mis en évidence l'influence de la longueur et surtout de la position de la chaîne grasse sur le squelette oligoglycérol, alors que la nature de la liaison chimique (ester ou éther) ne modifie pas le score d'irritation [27, 30].

\section{CONCLUSION}

Cette étude de la chimie des polyglycérols nous a amenés à préparer à l'échelle de plusieurs grammes des échantillons purs d'oligomères de glycérol de structure linéaire et cyclique ainsi qu'une sélection de tensioactifs qui n'avaient pour la plupart jamais été synthétisés sélectivement. Ces substances sont potentiellement des standards analytiques, utilisables comme étalons pour les mélanges conventionnels. Enfin, à travers les différentes évaluations réalisées, une première rationalisation des comportements de cette famille de composés (relations structure-activité) a pu être menée.

\section{Remerciements}

Cette étude de la chimie des polyglycérols a été réalisée grâce au financement de l'Union européenne par le biais du contrat FAIR DG XII n 97-1829. Remerciements également aux partenaires de ce projet européen, et particulièrement à Sylvain Claude, coordinateur du programme.

* Cet article est extrait d'un travail qui s'est vu décerner le prix de thèse 2001 de l'Association française pour l'étude des corps gras (AFECG).

\section{REFERENCES}

1. CLAUDE S (1999). Oleochemistry as an opportunity to consolidate the sustainable development of European oil crops - an updated prospect. OCL, $6: 418-27$.

2. KAUFMAN VR, GARTI N (1982). Organic reactions in emulsions - preparation of glycerol and polyglycerol esters of fatty acids by transesterification reaction. J Am Oil Chem Soc, 59 : 471-4.

3. CLAUDE S (1997). La valorisation du glycérol : quelques perspectives. OCL, $4: 250-2$.

4. SAHASRABUDHE MR (1967). Chromatographic analysis of polyglycerols and their fatty acid esters. J Am Oil Chem Soc, 376-8.

5. DE MEULENAER B, VANHOUTTE B, HUYGHEBAERT A (2000). Development of chromatographic method for determination of degree of polymerization of polyglycerols and polyglycerol fatty acid esters.

Chromatographia, $51: 44-52$.

6. GARTI N, ASARIN A, ZAIDMAN B (1981). Polyglycerol esters: optimization and techno-economic evaluation. J Am Oil Chem Soc, $58: 878-83$.

7. KUMAR NT, SASTRY YSR, LAKSHMINARAYANA G (1984). Analysis of polyglycerols by high-performance liquid chromatography. J Chromatogr, $298: 360-5$. 
8. ZHU Y, MASUYAMA A, KIRITO Y, OKAHARA M, ROSEN MJ (1992). Preparation and properties of glycerolbased double- or triple-chain surfactants with two hydrophilic ionic groups. J Am Oil Chem Soc, 69 : 626-32.

9. BABAYAN VK, McINTYRE RT (1971). Preparation and properties of some polyglycerol esters of short and medium chain length fatty acids. J Am Oil Chem Soc, 48 : 307-9.

10. FAIR DG XII n 97-1829. Polyglycerols chemistry, ecology and applications of polyglycerol Esters. Onidol, coordination du programme ; ENSCR : synthèse et analyse d'oligoglycérols linéaires et cycliques ; Icoa, Université d'Orléans : synthèse et analyse d'oligoglycérols ramifiés ; Université Complutense de Madrid : estérification enzymatique; Rhône-Poulenc : étude de la biodégradabilité ; Henkel KGaA : étude du pouvoir irritant, des propriétés tensioactives et de l'écotoxicité. 1997-2000.

11. McINTYRE RT (1979). Polyglycerol esters. J Am Oil Chem Soc, 56 : 835-40.

12. WRIGHT HJ, Du PUIS RN (1946). Diglycerol by a new ether synthesis. J Am Chem Soc, 68 : 446-8.

13. WITTCOFF H, ROACH JR, MILLER SE (1949). Polyglycerols. I. The identification of polyglycerol mixtures by the procedures of allylation and acetonation: isolation of pure diglycerol. J Am Oil Chem Soc, 71 : 2666-8.

14. ROACH JR, WITTCOFF H (1949). Polyglycerols III. Synthesis of triglycerol. J Am Chem Soc, 71 : 3944-6.

15. CASSEL S, DEBAIG C, BENVEGNU T, et al. (2001). Original synthesis of linear, branched and cyclic oligoglycerol standards. Eur J Chem, 875-96.

16. URATA K, TAKAISHI N (1994). The alkyl glycidyl ether as synthetic building blocks. J Am Oil Chem Soc, 71 : 1027-33.

17. WEBER WP, GOKEL GW (1977). Phase transfer catalysis in organic synthesis. Berlin : Springer-Verlag ; 280 p.

18. FREEDMAN HH, DUBOIS RA (1975). An improved Williamson ether synthesis using phase transfer catalysis. Tetrahedron Lett, 38 : 3251-4.

19. BRADSHAW JS, IZATT RM, BORDUNOV AV, ZHU CY, HATHAWAY JK (1996). Crown ethers. Compr Supramol Chem, $1: 35-95$.

20. PEDERSEN CJ (1967). Macrocyclic polyethers for complexing metals. J Am Chem Soc, 89 : 7017-36.

21. OUCHI M, INOUE Y, KANZAKI T, HAKUSHI T (1984). Molecular design of crown ethers. 1. Effects of methylene chain length: 15- to 17-crown-5 and 18- to 22-crown-6. J Org Chem, 49 : 1408-12.

22. COLLIE L, DENNESS JE, PARKER D, O'CAROLL F, TACHON C (1993). Synthesis of functionalised 12-, 13- and 14-membered crown ethers bearing exocyclic polymerisable groups and the binding properties and conductivity of their lithium doped polymers. J Chem Soc Perkin Trans, 2 : 1747-58.

23. GIBSON HW, BHEDA MC, ENGEN P, et al. (1994). Synthesis and characterization of large (30-60-membered) aliphatic crown ethers. J Org Chem, 59 : 2186-96.

24. HENKEL KGaA (1999). Primary monofatty acid esters of self condensation products of glycerol. DE : 199 : 49518-1. 
25. HENKEL KGaA (1999). Primary monoalkyl ethers of self condensation products of glycerol. DE : 199 : 590001.

26. PLUSQUELLEC D, ROULLEAU F, BERTHO F, LEFEUVRE M, BROWN E (1986). Chimie des sucres sans groupements protecteurs $-I$ - Estérification régiosélective de l'hydroxyle anomère du lactose, du maltose et du glucose. Tetrahedron, 42 : 2457-67.

27. DEBAIG C (2000). Synthèse de polyglycérols linéaires et cycliques. Tensioactifs polyglycérylés : synthèse et caractérisations. Thèse de doctorat de l'Université de Rennes I ; 183 p.

28. CHAIMBAULT P, CASSEL S, CLAUDE S, et al. (1999). Direct analysis of industrial oligoglycerols by liquid chromatography with evaporative light-scattering detection and mass spectrometry. Chromatographia, 50 : 239-42.

29. CASSEL S, CHAIMBAULT P, DEBAIG C, et al. (2001). Liquid chromatography of polyglycerol fatty esters and fatty ethers on porous graphitic carbon and octadecyl silica by using evaporative light scattering detection and mass spectrometry. J Chromatogr, A 95-106.

30. Travail en cours de publication. 


\section{Illustrations}<smiles>OCC(O)COCC(O)CO</smiles>

Diglyónols ramifíés<smiles>OCC(O)COC(CO)CO</smiles>

Diglyoérols orcliques<smiles>OCC1COCC(O)CO1</smiles>

Figure 1. Structures potentielles de diglycérols.

Tétraglycénol linéaire<smiles>COCC1CO1</smiles>

Synthons clés

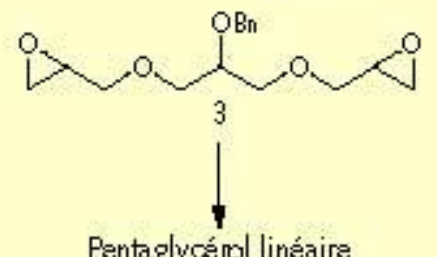

Figure 2. Voie d'accès aux oligoglycérols linéaires. 

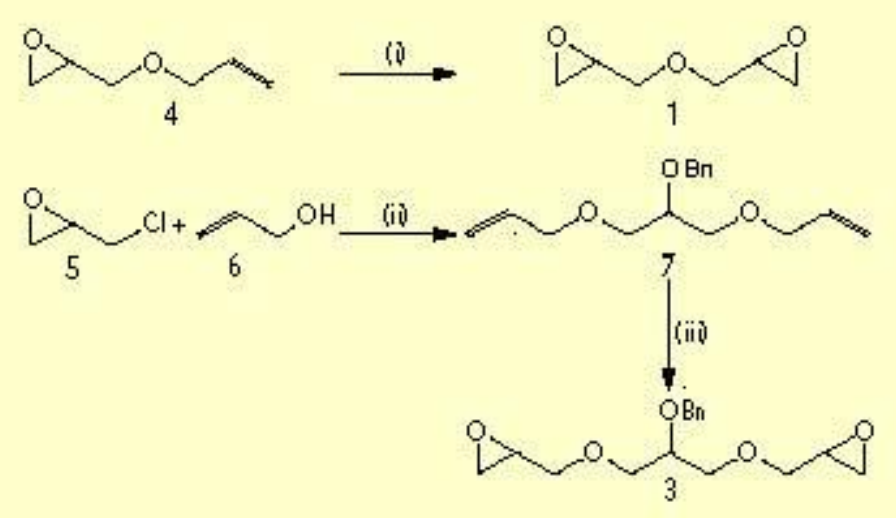

Figure 3. Synthèse des bis-époxydes 1 et 3.

(i) $m$ CPBA, $\mathrm{CH}_{2} \mathrm{Cl}_{2}, 94 \%$; (ii) a) NaOHaq $18 \mathrm{M}$, TBAB, $n$-hexane ; b) $\mathrm{BnBr}$, TBAB, THF 65

$\%$; (iii) $m C P B A, \mathrm{CH}_{2} \mathrm{Cl}_{2}, 63 \%$

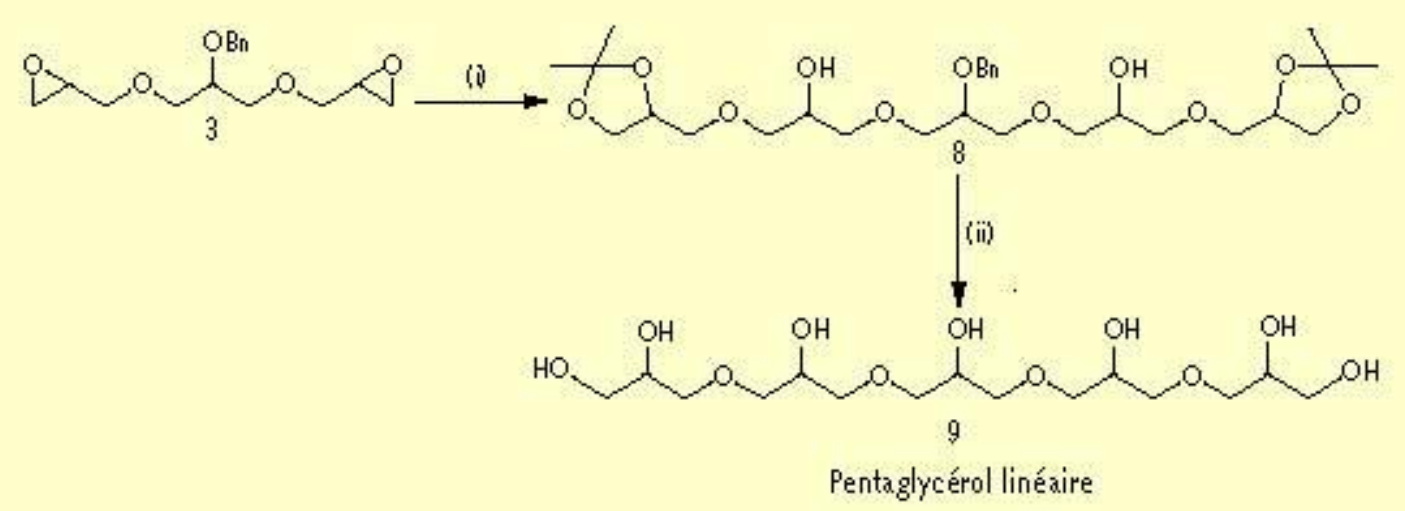

Figure 4. Obtention du pentaglycérol linéaire.

(i) Solkétal, NaOHaq, $50 \%$, Aliquat ${ }^{\circ}$, n-hexane, $53 \%$; (ii) $\mathrm{H}_{2}, \mathrm{Pd} / \mathrm{C}, \mathrm{MeOH}, 66 \%$. 


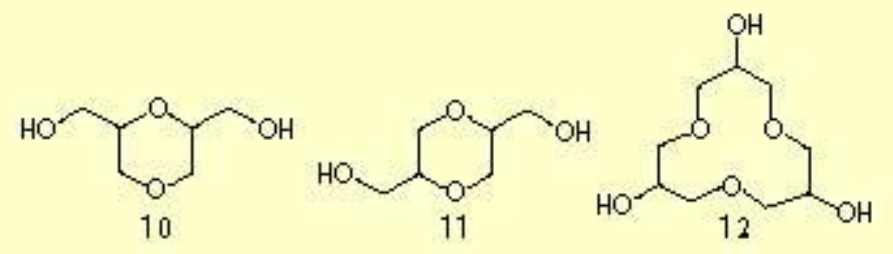

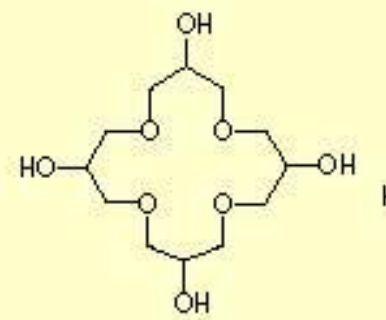

13

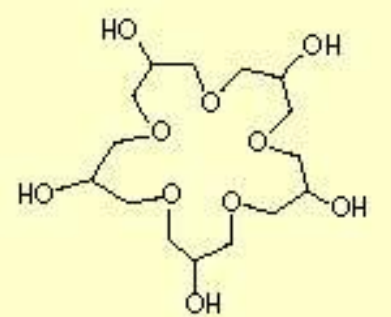

14

Figure 5. Structures cycliques synthétisées [15].
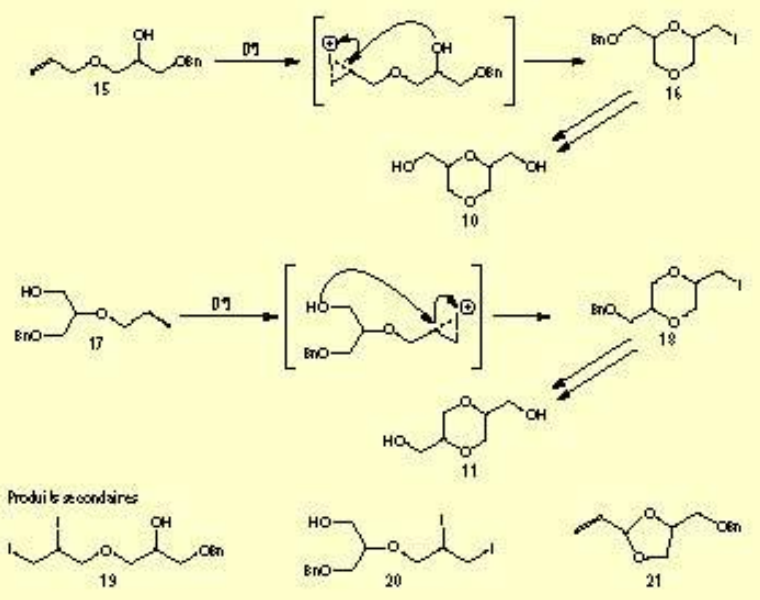

Figure 6. Schéma de préparation des diglycérols cycliques 10 et 11. 


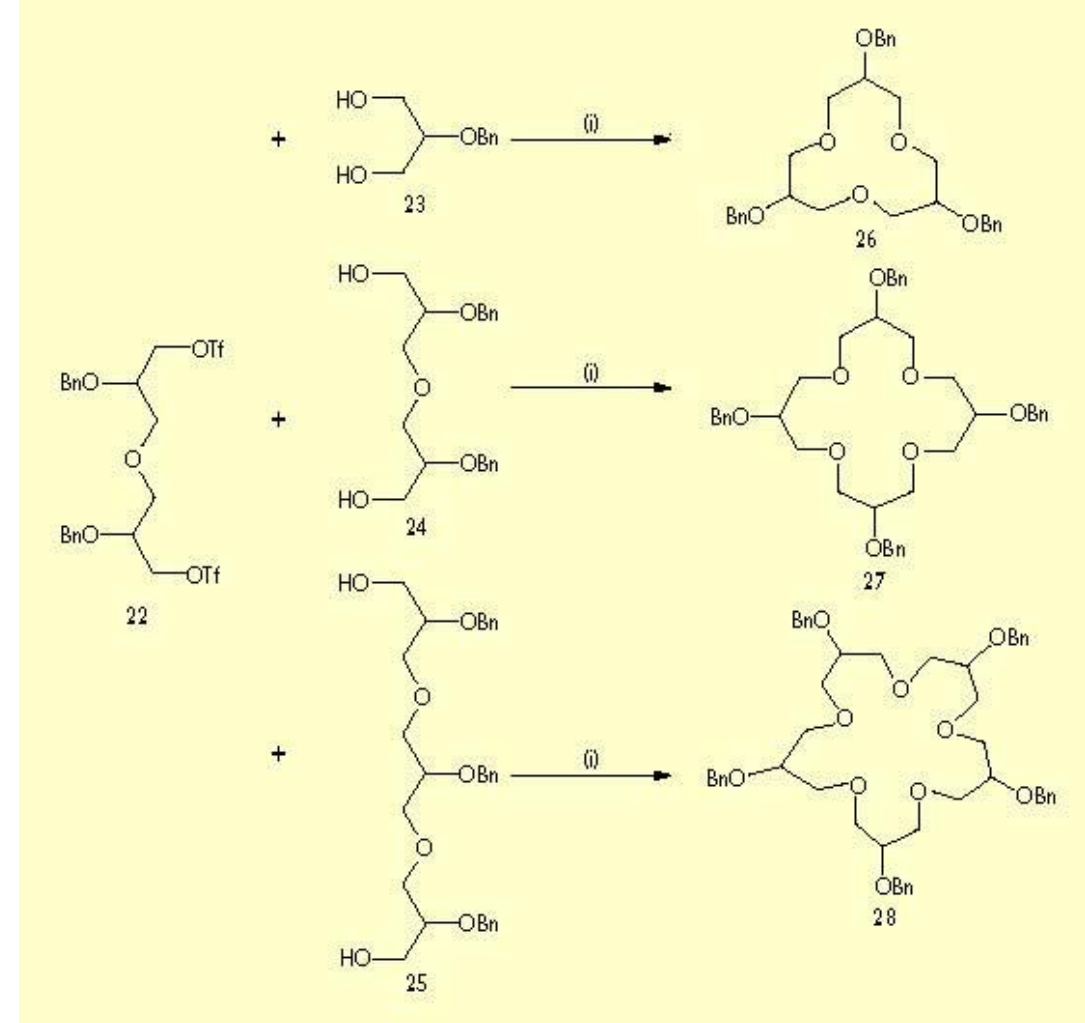

Figure 7. Synthèse des tri-, tétra- et pentaglycérols cycliques perbenzylés.

(i) $\mathrm{NaH}, \mathrm{THF}, 35 \%$.

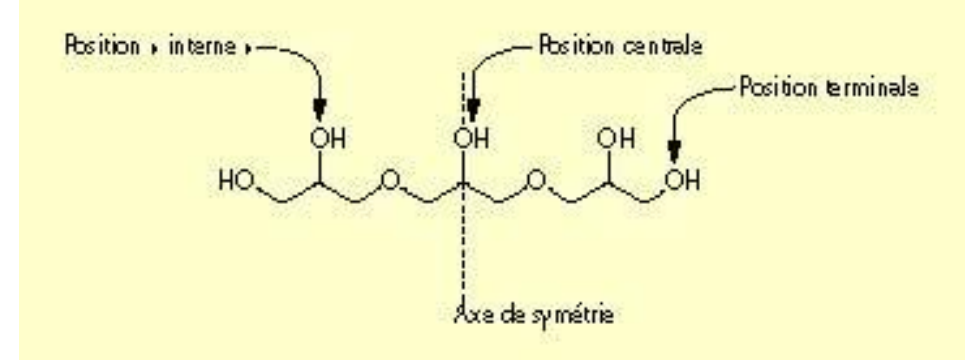

Figure 8. Tête polaire triglycérylée. 


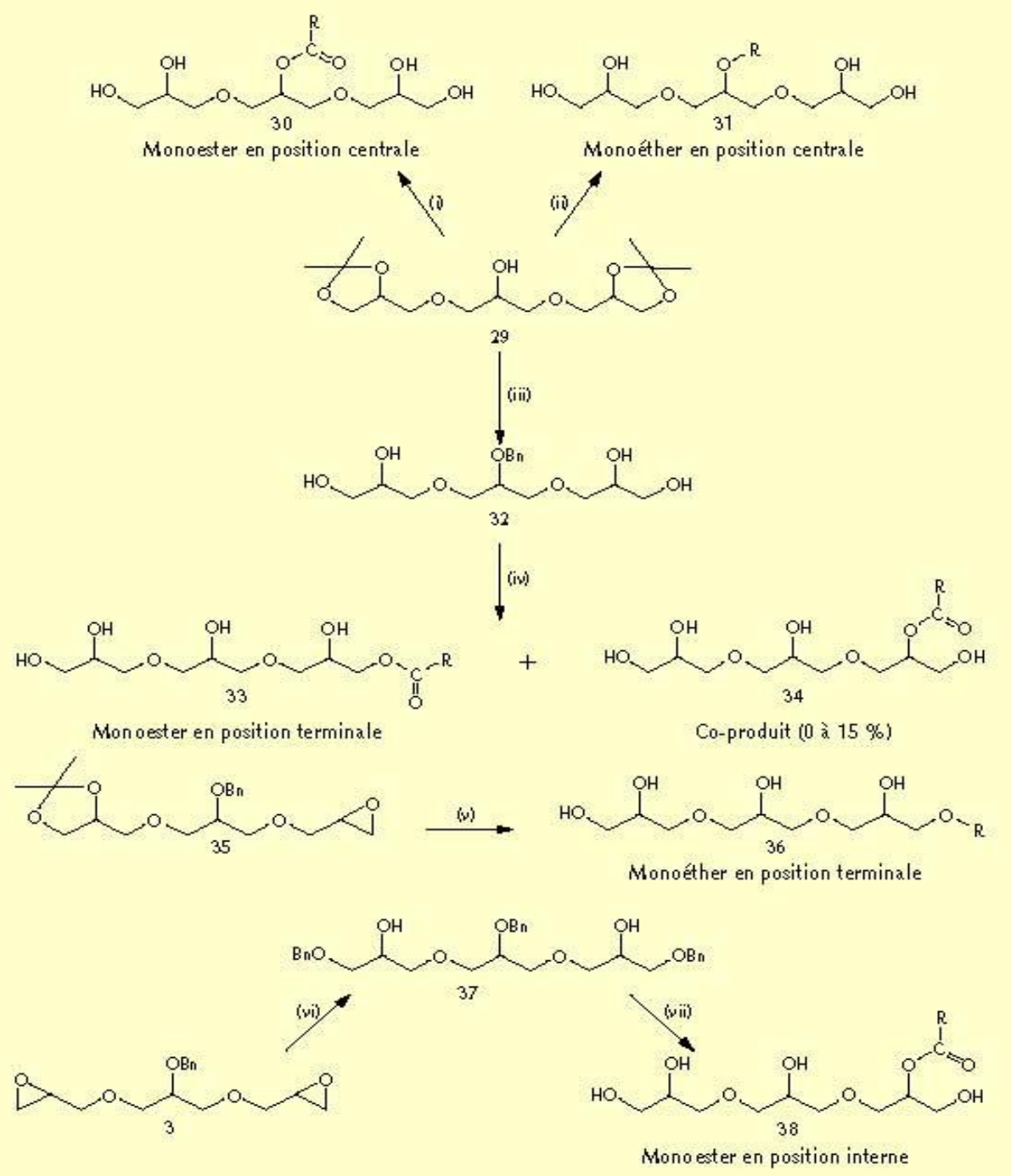

Figure 9. Schéma général de la préparation d'amphiphiles à partir du triglycérol linéaire.

(i) a) $\mathrm{RCOCl}, \mathrm{Pyr}, \mathrm{DMAP}, \mathrm{CH}_{2} \mathrm{Cl}_{2}$; b) Dowex $\mathrm{H}+\mathrm{MeOH}$; 54-62 \% ; (ii) a) R-Ots, $\mathrm{NaH}, \mathrm{DMF}$; b) Dowex $\mathrm{H}+\mathrm{MeOH}$; $55 \%$; (iii) a) $\mathrm{BnBr}, \mathrm{NaH}, \mathrm{TBAB}, \mathrm{THF}$; b) Dowex $\mathrm{H}+; 63 \%$; (iv)<smiles>[R]C(=O)N1CCCC1=S</smiles>

$\mathrm{NaH}, \mathrm{THF}$; 45-47\% ; b) $\mathrm{H}_{2} \mathrm{Pd} / \mathrm{C}, \mathrm{MeOH}, 90 \%$ ou $\mathrm{BCl}_{3} \cdot \mathrm{Sme}_{2}, \mathrm{CH}_{2} \mathrm{Cl}_{2}, 50 \%$ (cas $\mathrm{R}=$ C16:1)

(v) a) R-OH, $\mathrm{NaOH} 50 \%$ aq, TBAB, $n$-hexane, $53 \%$; b) $\mathrm{H}_{2}, \mathrm{Pd} / \mathrm{C}, \mathrm{MeOH}, 84 \%$; (vi) $\quad \mathrm{BnOH}, \quad \mathrm{NaH}, \quad 65 \quad \%$;

(vii) a) idem iv a), $55 \%$; b) $\mathrm{Pd}(\mathrm{II})$ acetate, $\mathrm{MeOH}, 81 \%$. 


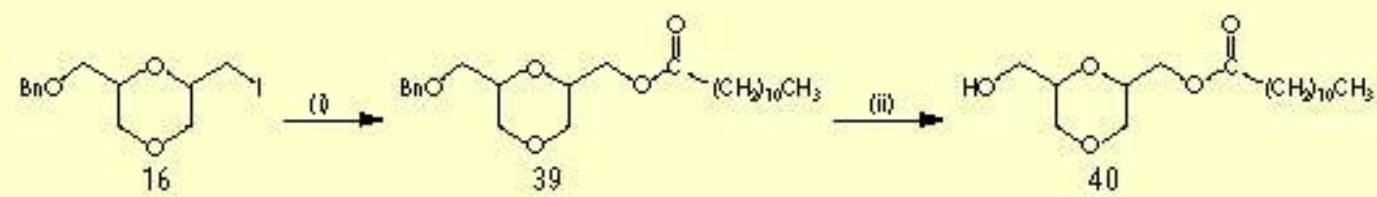

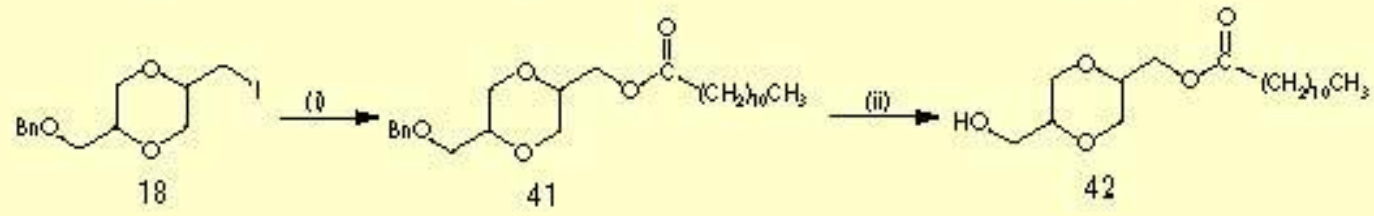

Figure 10. Formation des monolaurates de diglycérols cycliques.

(i) $\left(\mathrm{CH}_{3}-\left(\mathrm{CH}_{2}\right)_{10} \mathrm{COOH}, \mathrm{K}_{2} \mathrm{CO}_{3}\right.$, DMSO, $90{ }^{\circ} \mathrm{C}, 61$ à $70 \%$; (ii) $\mathrm{H}_{2}, \mathrm{Pd} / \mathrm{C}, \mathrm{MeOH}, 89$ à $98 \%$.

Tableau 1. Déplacements chimiques (di) ' $\mathrm{H}$ et ${ }^{3} \mathrm{C}\left(\mathrm{CD}_{3} \mathrm{OD}\right)$ caractéristiques des monoesters.<smiles>[R]C(=O)OC(I)C(O)COCC(O)C(C)C</smiles>

\begin{tabular}{|c|c|c|c|}
\hline & & $\delta(\mathrm{ppm})^{\mathrm{H}} \mathrm{H}$ & $\delta(\mathrm{ppm})^{13 \mathrm{C}}$ \\
\hline Ester en $\mathrm{Cl}$ & $\begin{array}{c}\mathrm{CH}_{2}-\mathrm{OCOR} \\
\mathrm{CH}-\mathrm{OH}(\mathrm{C} 2)\end{array}$ & $\begin{array}{c}4-4,2(2 \mathrm{H}) \\
3,95\end{array}$ & $\begin{array}{l}66,5 \\
69,5\end{array}$ \\
\hline Ester en C2 & $\begin{array}{c}\mathrm{CH}_{2}-\mathrm{OH}(\mathrm{Cl}) \\
\mathrm{CH}-\mathrm{OCOR}\end{array}$ & $\begin{array}{l}3,7-3,8 \\
5,1(1 \mathrm{H})\end{array}$ & $\begin{array}{l}61,7 \\
74,5\end{array}$ \\
\hline Ester en C6 & $\begin{array}{c}\mathrm{CH}_{2}-\mathrm{OH}(\mathrm{Cl}) \\
\mathrm{CH}-\mathrm{OH}(\mathrm{C} 2) \\
\mathrm{CH}-\mathrm{OCOR}\end{array}$ & $\begin{array}{c}3,3-3,5 \\
3,65 \\
5,05(1 \mathrm{H})\end{array}$ & $\begin{array}{l}64,4 \\
72,2 \\
72,8\end{array}$ \\
\hline
\end{tabular}


Tableau 2. Amphiphiles syrthétisés [27].

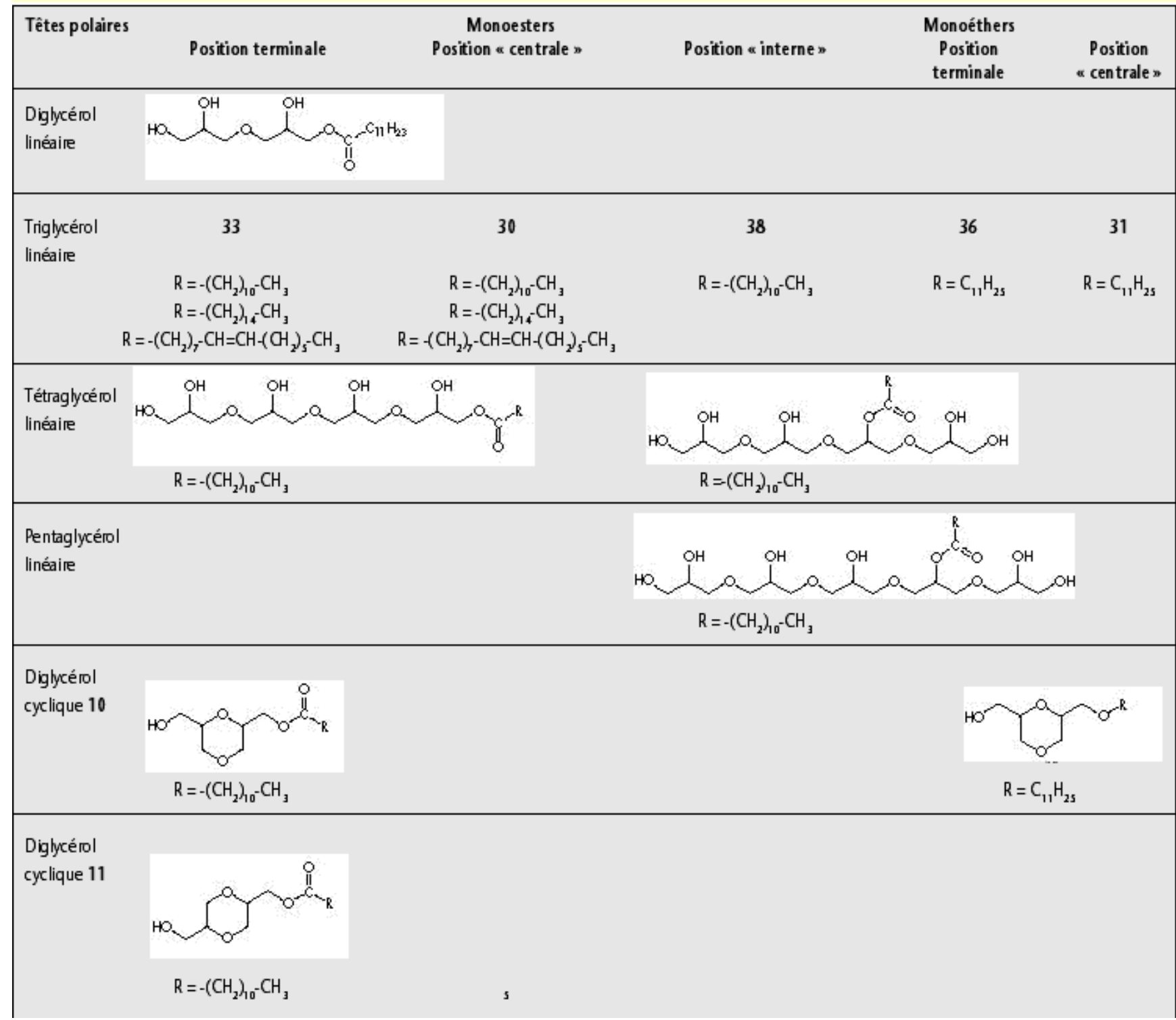

